\title{
Absolute Neutrophil Count in the Peripheral Blood Predicts Prognosis in Lung Cancer Patients Treated with Anlotinib
}

\author{
Rong Chen ${ }^{1,2, *}$ \\ Fang-Ying Lu ${ }^{1,2, *}$ \\ Bing Liu ${ }^{1,2}$ \\ Jingwen Huang $\mathbb{D}^{1,2}$ \\ Min Zhou ${ }^{1,2}$ \\ Ranran Dai ${ }^{1,2}$ \\ Yi Guo ${ }^{1,2}$
}

'Department of Respiratory and Critical Care Medicine, Ruijin Hospital, Shanghai Jiao Tong University School of Medicine, Shanghai, People's Republic of China; ${ }^{2}$ Institute of Respiratory Diseases, Shanghai Jiao Tong University School of Medicine, Shanghai, People's Republic of China

*These authors contributed equally to this work
Correspondence: Yi Guo; Ranran Dai Department of Respiratory and Critical Care Medicine, Ruijin Hospital, Shanghai Jiao Tong University School of Medicine, No. 197, Rui Jin 2nd Road, Shanghai, 200025, People's Republic of China

Tel +86 I3। 6622 I556;

$\mathrm{Tel}+86$ I3| 6622 I556

Fax +86 2I 64674301

Email guoyi62।@qq.com;

drrlII54@rjh.com.cn
Purpose: Anlotinib is a multi-targeted tyrosine kinase inhibitor that inhibits tumor angiogenesis and cell proliferation. It is widely used as a third-line therapy for lung cancer. However, reliable prognostic biomarkers for predicting the efficacy of anlotinib are lacking. We conducted a retrospective study to investigate the prognostic value of serological inflammatory biomarkers in anlotinib treatment.

Patients and Methods: Patients with advanced lung cancer treated with anlotinib monotherapy were enrolled. Cox regression was conducted to analyze the significant factors related to progression-free survival (PFS) and overall survival (OS). The objective response rate (ORR) was compared based on the median cut-off value of the significant inflammation index. Meanwhile, we created survival curves to compare the two groups and performed receiver operating characteristic curve analysis to assess the predictive ability of the inflammation index. Results: Among a total of 71 patients, the median PFS was 5.5 months and the median OS was 9.5 months. The ORR and disease control rate were $16.9 \%$ and $84.5 \%$, respectively. According to univariate and multivariate analyses, absolute neutrophil count (ANC) was the only indicator associated with both PFS (hazard ratio $[\mathrm{HR}]=1.095,95 \%$ confidence interval [CI] 1.030-1.163, $\mathrm{P}=0.003)$ and $\mathrm{OS}(\mathrm{HR}=1.057,95 \% \mathrm{CI} 1.003-1.113, \mathrm{P}=0.037)$. In the group with $\mathrm{ANC} \geq 4.58$, the $\mathrm{ORR}$ was relatively lower $(8.1 \%$ vs $26.5 \%, \mathrm{P}=0.057$ ), but not statistically significant; PFS and OS were relatively shorter (median PFS 5.0 [95\% CI 4.4-9.6] vs 7.0 months [95\% CI 4.4-5.7], $\mathrm{P}=0.024$ and median OS 7.3 [95\% CI 4.7-10.0] vs 17.6 months [95\% CI 12.3-22.9], $\mathrm{P}<0.001$ ). ANC had a relatively high discriminatory ability to predict 10-month survival, with an area under the curve of 0.729 , sensitivity of $82.5 \%$, and specificity of $67.7 \%$.

Conclusion: Elevated pre-treatment ANC was associated with a poor prognosis. Patients with lower peripheral blood levels of ANC might benefit from anlotinib.

Keywords: lung cancer, anlotinib, absolute neutrophil count, progression-free survival, overall survival

\section{Introduction}

Lung cancer is the leading cause of cancer-related mortality worldwide. According to Global Cancer Statistics in 2020, there were an estimated 2.2 million new lung cancer cases and 1.8 million deaths, accounting for about $11.4 \%$ of newly diagnosed cancers and $18.0 \%$ of deaths. ${ }^{1}$ In terms of histopathology, lung cancer is usually classified as non-small cell lung cancer (NSCLC) and small cell lung cancer (SCLC). In case of NSCLC, approximately $75 \%$ of the patients are already at an 
advanced stage upon diagnosis, with a 5-year survival rate of $<15 \%{ }^{2}$ SCLC is characterized by an aggressive clinical course, with a 5-year overall survival (OS) of 20-25\% for early-stage disease and $2 \%$ for extended disease. ${ }^{3}$ Currently, in addition to conventional chemotherapy, the emergence of targeted therapy based on genomic medicine and immunotherapy based on immunological checkpoint inhibitors has significantly improved the quality of life and survival time of patients. ${ }^{4}$ Antiangiogenic drugs are also considered to have promising therapeutic effects, especially in patients who cannot tolerate chemotherapy, those with untreatable gene mutations or with pathologically confirmed immunologically-cold tumors. ${ }^{5}$

Anlotinib is a newly developed oral multi-targeted tyrosine kinase receptor inhibitor that targets the vascular endothelial growth factor receptor (VEGFR) 1-3, c-Kit, platelet-derived growth factor receptor- $\alpha$, and fibroblast growth factor receptor $1-4$. It can inhibit both tumor angiogenesis and tumor cell proliferation. ${ }^{6,7}$ In recent years, two randomized, double-blind, placebo-controlled, multicenter, Phase II/III trials comparing the efficacy and safety of anlotinib with that of placebo in refractory NSCLC patients who progressed after at least two lines of prior treatments have revealed its efficacy. ${ }^{8,9}$ In China, anlotinib is one of the most common antiangiogenic drugs used in lung cancer treatment. However, not all patients with advanced lung cancer will benefit from anlotinib and the predictive biomarkers to select suitable patients are insufficient. Although clinical trials have identified some biomarkers as predictors of anlotinib activity, such as activated circulating endothelial cells, epidermal growth factor receptor (EGFR)-sensitizing mutations, and the T790M mutation, ${ }^{10}$ these markers are not universal and are expensive. Hence, there is a considerable need to identify feasible prognostic biomarkers for anlotinib therapy.

Peripheral blood-based inflammatory parameters, such as C-reactive protein (CRP), absolute neutrophil count (ANC), and absolute lymphocyte count (ALC) are readily available in the clinic. These parameters and their composite indicators, such as neutrophil-to-lymphocyte ratio (NLR) and platelet-to-lymphocyte ratio (PLR), have shown prognostic value in a spectrum of malignancies and could predict outcomes of patients with platinumbased chemotherapy, nivolumab treatment, and bevacizumab therapy. ${ }^{11-16}$ To date, no study has focused on the correlation between peripheral blood-based inflammatory parameters and the therapeutic efficacy of anlotinib treatment. Thus, we conducted a retrospective study to explore the predictive value of candidate inflammatory biomarkers in patients with lung cancer treated with anlotinib.

\section{Materials and Methods Study Design and Patient Selection}

A total of 181 patients who had advanced lung cancer and were treated with anlotinib (one cycle of $12 \mathrm{mg}$ daily for 14 days, then discontinued for 7 days, and repeated every 21 days) in Ruijin Hospital between July 2018 and February 2020 were screened. All patients were diagnosed with lung cancer according to histopathological criteria (World Health Organization, 2015). Patients in advanced or recurrent stage IIIA-B/IV based on the TNM classification (version 7) with an Eastern Cooperative Oncology Group performance status (ECOG PS) of 0-1 were enrolled initially. Patients with concomitant infections, including human immunodeficiency virus and hepatitis, or those receiving systemic steroids before anlotinib treatment were excluded. Finally, 71 patients with complete clinical data and treatment with anlotinib monotherapy were included in the study. From the first dose of anlotinib, all patients were followed up for at least 10 months. Since this was a retrospective study that only analyzed the clinical data in the patient's medical records, informed consent was formally waived by the Ethics Committee of Ruijin Hospital. This study was conducted in accordance with the Declaration of Helsinki, and all patient information was kept confidential.

\section{Data Collection}

We gathered data on demographics, clinicopathological characteristics, distant metastases, gene mutations, and previous treatment lines from the electronic medical records. Complete blood cell counts were performed at baseline within a week prior to the first dose of anlotinib. We collected baseline data on serological markers of inflammation, including CRP, ANC, and ALC.

\section{Study Assessments}

Therapeutic effects were assessed using the Response Evaluation Criteria in Solid Tumors version 1.1, by performing computed tomography scans and nuclear magnetic resonance imaging every 2 months or when the clinical symptoms worsened. Progression-free survival (PFS) was defined as the duration from the beginning of anlotinib administration to tumor progression or death. Objective tumor responses consisted of complete response (CR), partial response (PR), stable disease (SD), and 
progressive disease (PD). The objective response rate (ORR) included the $\mathrm{CR}$ and $\mathrm{PR}$, and the disease control rate (DCR) included CR, PR, and SD. The 10-month survival was defined as the time from the first day of anlotinib treatment to death or the last follow-up of 10 months.

\section{Statistical Analysis}

Categorical variables were reported as frequencies with percentages, and continuous variables were described as medians with interquartile range (IQR). Significant factors related to PFS and OS were estimated using univariate and multivariate Cox regression analyses. According to the median ANC, patients were divided into relatively high and low level groups. The Log rank test was used for the analysis of PFS and OS and survival curves were created using the Kaplan-Meier method. The response to anlotinib between the two groups was compared using the Pearson $\chi^{2}$ or Fisher's exact test. Receiver operating characteristic (ROC) curves and the area under the curve (AUC) were used to assess the ability of ANC to predict the 10-month survival. The level of significance for all statistical tests was set at a two-sided P value of 0.05 . Statistical analyses were performed using the SPSS Version 25.0 statistical software (IBM Corporation, Armonk, NY, USA).

\section{Results}

\section{Patient Characteristics}

Based on the inclusion and exclusion criteria, 71 patients with relatively complete clinical, pathological and follow- up data were included in the study (Figure 1). The average age of the patients was $68 \pm 9$ years with a male predominance $(71.8 \%)$. A total of 45 patients $(63.4 \%)$ had a smoking history and 70 patients (79.5\%) had an ECOG PS of 0 . Overall, 49 patients $(69.0 \%)$ had adenocarcinoma, $14(57.7 \%)$ had squamous cell carcinoma, 7 (9.9\%) had small cell lung cancer, and 1 (1.4\%) had another histologic type. Brain metastasis accounted for $11.3 \%$ of the metastatic sites, liver metastasis for $2.8 \%$, and bone metastasis for $28.2 \%$. Additionally, 61 patients $(85.9 \%)$ had lymphatic metastasis. Stage IV disease was present in 56 patients (78.9\%), with $12(16.9 \%)$ having more than one metastatic site. EGFR mutations were identified in 7 patients $(9.9 \%)$. Regarding treatment, 54 patients $(76.0 \%)$ received palliative chemotherapy, $18(25.4 \%)$ received radiation therapy, $27(38.0 \%)$ received targeted therapy, and $1(1.4 \%)$ received PD-1 inhibitor treatment prior anlotinib treatment. More than three prior lines of therapy were noted in 28 patients $(39.4 \%)$. The median CRP, ANC, and ALC were $12.0 \mathrm{mg} / \mathrm{L}$ (IQR, 3.0-48.3), 4.6 (IQR, 3.7-6.3), and 1.3 (IQR, 0.9-1.7), respectively. The characteristics of all patients are listed in Table 1.

\section{Survival Outcomes}

For the patients included in the analysis, the median PFS (mPFS) was 5.5 months (IQR, 4.0-8.0) and median OS (mOS) was 9.5 months (IQR, 6.0-3.4). According to univariate analysis, number of prior lines of therapy $>3$ (hazard ratio $[\mathrm{HR}]=1.735$; 95\% confidence interval $[\mathrm{CI}]$ 1.013-2.974, $\mathrm{P}=0.045)$ and higher levels of $\mathrm{ANC}(\mathrm{HR}=1.105 ; 95 \% \mathrm{CI}$

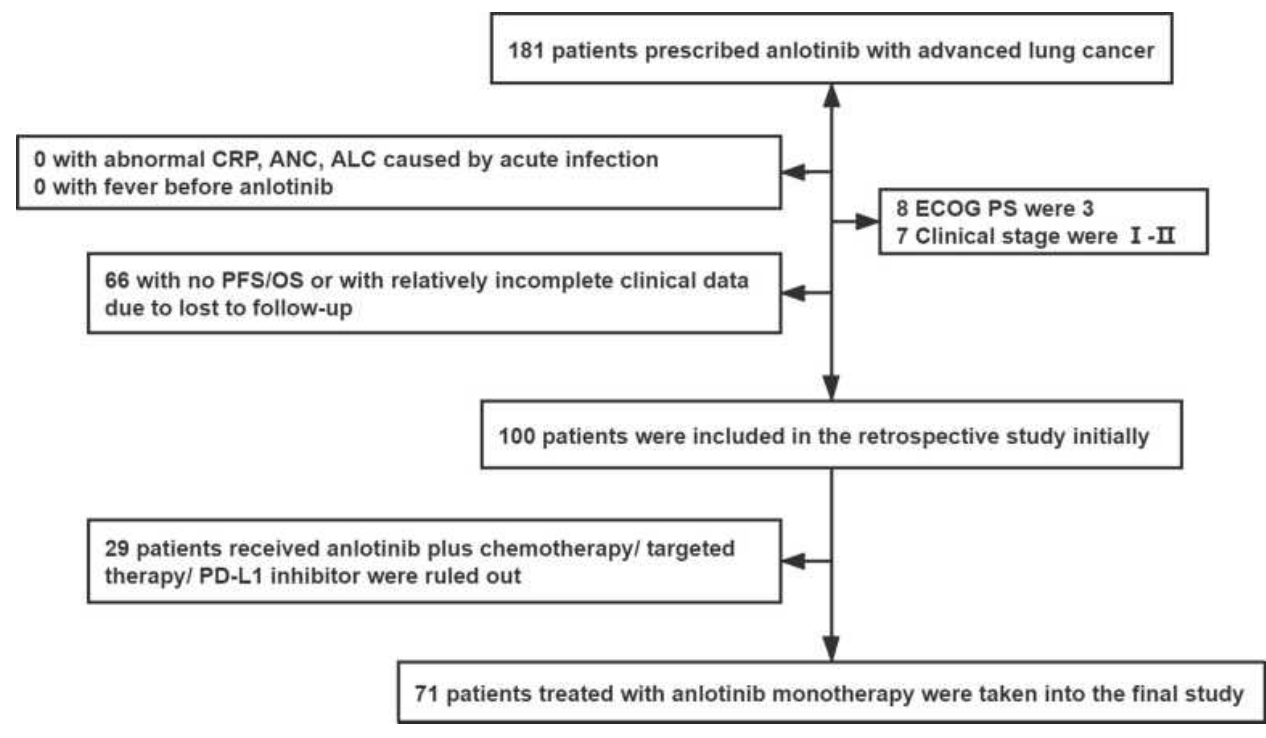

Figure I Flowchart of the study participants. 
Table I Baseline Characteristics of Study Participants

\begin{tabular}{|l|l|l|}
\hline Gender, N (\%) & Male & $5 \mathrm{I}(7 \mathrm{I} .8)$ \\
& Female & $20(28.2)$ \\
\hline $\begin{array}{l}\text { Age at start anlotinib } \\
\text { (years) }\end{array}$ & Average SSD & $68 \pm 9$ \\
\hline Tobacco, N (\%) & Never smoker & $26(36.6)$ \\
& Current smoker & $38(53.5)$ \\
$7(9.9)$
\end{tabular}

Note: ${ }^{a}$ Data were missing in three patients.

Abbreviations: EGFR, epidermal growth factor receptor; PD-I, programmed cell death protein-I; CRP, C-reactive protein; ANC, absolute neutrophil count; ALC, absolute lymphocyte count; SD, standard deviation; IQR, interquartile range.

$1.044-1.170, \mathrm{P}=0.001)$ were associated with a shorter PFS, while age $\geq 65$ years $(\mathrm{HR}=1.881 ; 95 \%$ CI $1.041-3.397$, $\mathrm{P}=0.036)$, tobacco use $(\mathrm{HR}=1.854 ; 95 \%$ CI 1.012-3.398, $\mathrm{P}=0.046)$, and higher $\mathrm{ANC}(\mathrm{HR}=1.070 ; 95 \% \mathrm{CI} 1.023-1.121$, $\mathrm{P}=0.004$ ) were associated with a shorter OS. We fitted multivariable Cox regression models using age $\geq 65$, tobacco use, number of prior lines of therapy, and ANC. Ultimately, only high ANC values were found to contribute significantly to the prediction of PFS $(\mathrm{HR}=1.095,95 \%$ CI $1.030-1.163$; $\mathrm{P}=0.003)$ and $\mathrm{OS}(\mathrm{HR}=1.057$, 95\% CI 1.003-1.113; $\mathrm{P}=0.037$ ). The univariate and multivariate Cox analyses are shown in Tables 2 and 3.

\section{Co-Relation Between ANC and Prognosis}

Regarding the result of the multivariable analysis, ANC was associated with the PFS and OS in patients treated with anlotinib. Therefore, we divided the patients into two groups according to median ANC values for further verification. There were no statistical differences in the demographics and clinical characteristics between the two groups, except for the ECOG PS (Supplementary Table 1). According to the assessment, the responses among the patients treated with anlotinib were PR $(n=12), \operatorname{SD}(n=48)$, and PD $(n=11)$. The ORR and DCR were $16.9 \%$ and $84.5 \%$, respectively. Higher baseline ANC (ANC $\geq 4.58$ ) was associated with a lower ORR, although there were no statistically significant differences between the two groups $(26.5 \%$ vs $8.5 \%, \mathrm{P}=0.057)$. Using the Log rank test, we also found that $\mathrm{ANC} \geq 4.58$ demonstrated inferior mPFS (5.0 [95\% CI 4.4-5.7] vs 7.0 months [95\% CI 4.4-9.6], $\mathrm{P}=0.024$; Figure 2) and $\operatorname{mOS}(7.3$ [95\% CI 4.7-10.0] vs 17.6 months [95\% CI 12.3-22.9], $\mathrm{P}<0.001$; Figure 3). The detailed analysis is shown in Table 4.

\section{Predictive Ability of ANC}

To better analyze the predictive ability of ANC, we used "10month survival" as the outcome to establish the predictive model. Based on ROC curve analysis (Figure 4), the AUC for ANC was 0.729 (95\% CI 0.603-0.854; $\mathrm{P}=0.001)$. With an optimal cut-off of 4.32 , the sensitivity and specificity for predicting 10-month mortality were $82.5 \%$ and $67.7 \%$, respectively.

\section{Discussion}

This was a retrospective study that aimed to determine the role of some inflammatory parameters in the peripheral blood in predicting the survival of patients with lung cancer who received anlotinib therapy. According to the results of univariate and multivariate analyses, $\mathrm{ANC}$ was related to the patient outcome. A higher baseline ANC was significantly associated with a shorter PFS and OS. ANC may also be a predictive biomarker for 10-month mortality. 
Table 2 Univariate Cox Regression Analyses of PFS and OS

\begin{tabular}{|c|c|c|c|c|c|c|}
\hline \multirow[t]{2}{*}{ Variable } & \multicolumn{3}{|l|}{ PFS } & \multicolumn{3}{|l|}{ os } \\
\hline & HR & $95 \% \mathrm{Cl}$ & $P$ value & HR & $95 \% \mathrm{Cl}$ & $P$ value \\
\hline Age $\geq 65$ (vs Age $<65$ years old $)$ & 1.182 & $0.696-2.007$ & 0.536 & 1.881 & $1.041-3.397$ & $0.036 *$ \\
\hline Male (vs female) & 1.264 & $0.708-2.256$ & 0.428 & 1.312 & $0.694-2.482$ & 0.403 \\
\hline Squamous (vs other) & 1.149 & $0.607-2.174$ & 0.669 & 1.504 & $0.76 \mathrm{I}-2.974$ & 0.240 \\
\hline Adenocarcinoma (vs other) & 1.009 & $0.577-1.765$ & 0.974 & 0.816 & $0.449-1.484$ & 0.506 \\
\hline ECOG PS at treatment start & 1.085 & $0.614-1.917$ & 0.779 & 1.573 & $0.895-2.766$ & 0.115 \\
\hline Tobacco use (vs none) & 1.206 & $0.703-2.068$ & 0.497 & 1.854 & $1.012-3.398$ & $0.046 *$ \\
\hline Liver metastasis (vs none) & 0.909 & $0.125-6.608$ & 0.925 & 2.440 & $0.586-10.154$ & 0.220 \\
\hline Skeletal metastasis (vs none) & $1.05 \mathrm{I}$ & $0.593-1.861$ & 0.865 & 0.799 & $0.42 I-1.514$ & 0.491 \\
\hline Brain metastasis (vs none) & 0.539 & $0.213-1.364$ & 0.192 & 0.607 & $0.236-1.562$ & 0.300 \\
\hline Lymphatic metastasis (vs none) & 0.674 & $0.339-1.339$ & 0.260 & 1.314 & $0.557-3.095$ & 0.533 \\
\hline IV Stage (vs IIIA-IIIB Stage) & 0.824 & $0.443-1.53 \mid$ & 0.540 & 0.638 & $0.331-1.229$ & 0.179 \\
\hline EGFR mutation (vs non-EGFR mutation) & 1.395 & $0.629-3.096$ & 0.413 & 0.902 & $0.356-2.286$ & 0.829 \\
\hline No. of previous treatment lines $>3$ (vs $\leq 3$ ) & 1.735 & $1.013-2.974$ & $0.045^{*}$ & 1.112 & $0.634-1.950$ & 0.710 \\
\hline Previous chemotherapy (vs with no previous therapy) & $\mathrm{I} .30 \mathrm{I}$ & $0.654-2.587$ & 0.453 & 0.788 & $0.386-1.610$ & 0.513 \\
\hline Previous targeted therapy (vs with no previous therapy) & 1.463 & $0.79 I-2.706$ & 0.226 & 1.835 & $0.928-3.626$ & $0.08 I$ \\
\hline Previous targeted therapy (vs with no previous therapy) & 1.376 & $0.798-2.37 \mid$ & 0.251 & 1.120 & $0.619-2.027$ & 0.708 \\
\hline Previous PD-I inhibitor (vs with no previous therapy) & $1.58 \mathrm{I}$ & $0.216-11.583$ & 0.652 & 2.658 & $0.357-19.77$ & 0.340 \\
\hline $\mathrm{CRP}^{\mathrm{a}}$ & 1.002 & $0.997-1.006$ & 0.479 & 1.003 & $0.998-1.007$ & 0.250 \\
\hline ANC & 1.105 & $1.044-1.170$ & $0.001 *$ & 1.070 & $1.023-1.121$ & $0.004 *$ \\
\hline ALC & $0.84 I$ & $0.567-1.249$ & 0.391 & 0.862 & $0.553-1.346$ & 0.515 \\
\hline
\end{tabular}

Note: ${ }^{a}$ Data were missing in three patients. $* p<0.05$.

Abbreviations: EGFR, epidermal growth factor receptor; PD-LI, programmed cell death protein ligand-I; CRP, C-reactive protein; ANC, absolute neutrophil count; ALC, absolute lymphocyte count; HR, hazard ratio; $\mathrm{Cl}$, confidence interval.

Table 3 Multivariate Cox Regression Analysis of PFS and OS

\begin{tabular}{|l|l|l|l|l|l|l|}
\hline \multirow{2}{*}{ Variable } & \multicolumn{2}{l|}{ PFS } & \multicolumn{2}{l|}{ OS } \\
\cline { 2 - 7 } & HR & $\mathbf{9 5 \% ~ C l}$ & P value & HR & $\mathbf{9 5 \% ~ C l}$ & P value \\
\hline Age $\geq 65$ (vs Age $<65$ years old) & 0.986 & $0.564-1.722$ & 0.960 & 1.560 & $0.846-2.877$ & 0.154 \\
Tobacco use (vs none) & 1.174 & $0.680-2.027$ & 0.565 & 1.745 & $0.946-3.218$ & 0.075 \\
No. of previous treatment lines $>3$ (vs $\leq 3)$ & 1.523 & $0.870-2.669$ & 0.141 & 0.890 & $0.486-1.629$ & 0.706 \\
ANC & 1.095 & $1.030-1.163$ & $0.003^{*}$ & 1.057 & $1.003-1.113$ & $0.037 *$ \\
\hline
\end{tabular}

Notes: Cox proportional hazard models using PFS and OS as a timescale to estimate hazard ratios (HRs) and $95 \%$ confidence intervals $(\mathrm{Cls})$. ${ }_{p}<<0.05$.

Anlotinib is a type of antiangiogenetic therapy that plays a critical role in the alleviation of the proliferation and invasion of malignant tumor cells. In the current study, the median PFS and OS were 5.5 months (IQR, 4.0-8.0) and 9.5 months (IQR, 6.0-13.4), respectively, which were in line with the results from ALTER 0303 trial and some other retrospective studies. ${ }^{9,17}$ Moreover, considerable improvements in the ORR (16.9\%) and DCR (84.5\%) were also observed in our study, which indicated that patients with advanced lung cancer could derive the clinical benefit of anlotinib in the real world.
With regard to the prognosis of lung cancer patients on anlotinib therapy, the potential predictive biomarkers are limited and insufficient. A recent retrospective study preliminarily found that tumor cavitation was an independent factor for predicting better PFS, but only a small number of patients with extensive-stage SCLC was included. ${ }^{18}$ Another large-scale study collected data from the ALTER0303 trial and concluded that baseline characteristics such as elevated thyroid-stimulating hormone, blood glucose, triglyceride levels, and hypertension were protective factors of prognosis in refractory NSCLC patients 


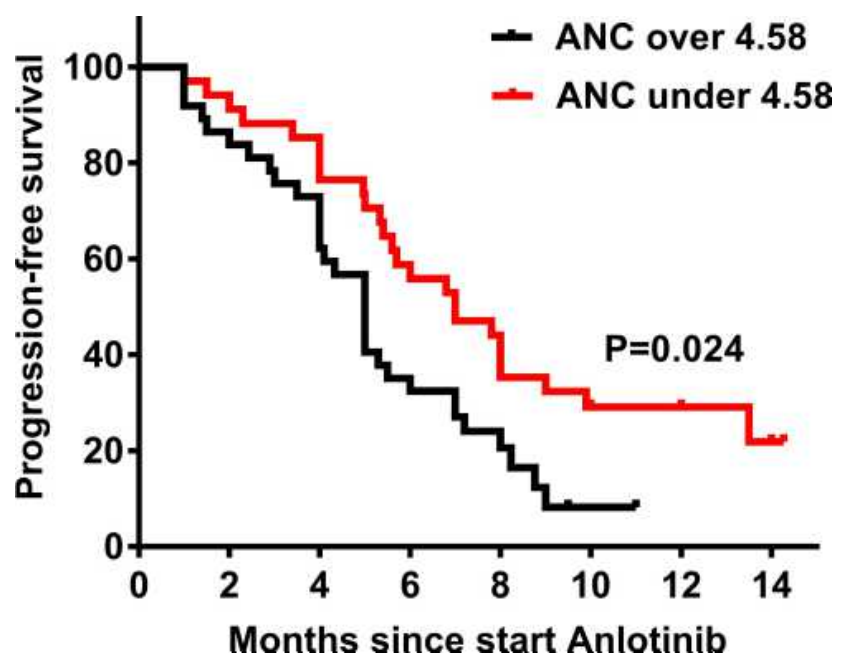

Figure 2 Progression-free survival (PFS) according to absolute neutrophil count (ANC). In the group with ANC $\geq 4.58$, the median PFS (mPFS) is 7.0 months, and the $95 \%$ confidence interval $(\mathrm{Cl})$ is $4.4-9.6$ months; In the group with $\mathrm{ANC}<4.58$, the mPFS is 5.0 months, and the $95 \% \mathrm{Cl}$ is $4.4-5.7$ months. $\mathrm{P}$ value is 0.024 .

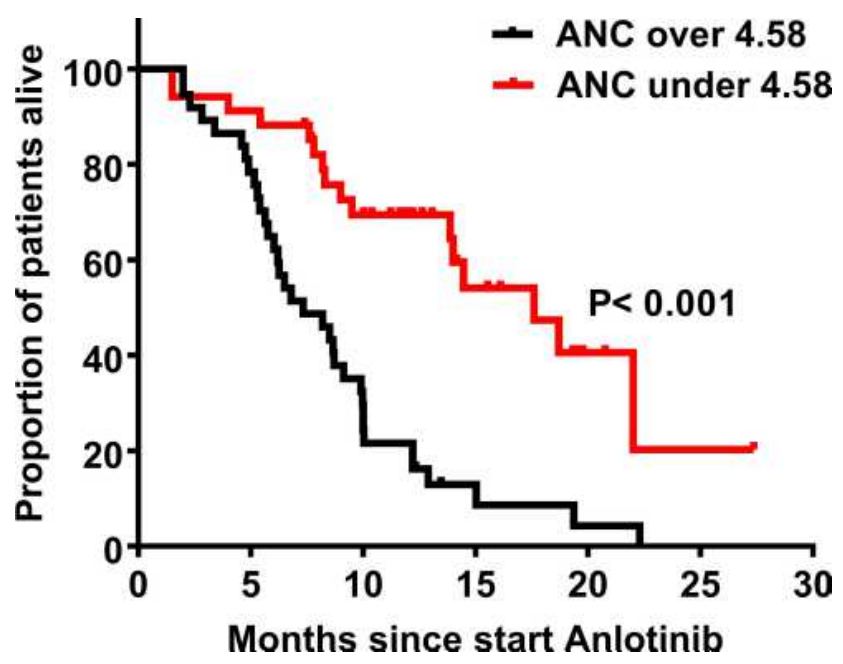

Figure 3 Overall survival (OS) according to ANC. In the group with ANC $\geq 4.58$, the median OS (mOS) is 17.6 months, and the $95 \% \mathrm{Cl}$ is $12.3-22.9$ months; In the group with ANC < 4.58, the mOS is 7.3 months, and the $95 \% \mathrm{Cl}$ is $4.7-10.0$ months. $P$ value is less than 0.001 .

receiving anlotinib. ${ }^{19}$ This study also showed that a high post-therapeutic peripheral blood granulocyte/lymphocyte ratio was an independent risk factor for PFS and OS, which attracted our attention.

Neutrophils are the most abundant type of innate immune cells. Accumulating evidence indicates that neutrophils play an important role in tumor proliferation, aggressiveness, and dissemination, as well as in immune suppression, through their interaction with cancer and immune cells in the blood and tumor microenvironment. ${ }^{20}$ Moreover, ANC has always been considered as a biomarker for the prognosis of lung
Table 4 ORR, PFS and OS According to Median ANC

\begin{tabular}{|l|l|l|l|}
\hline ANC & N for CR+PR & Percentage (\%) & P value \\
\hline$<4.58$ & 9 & 26.5 & \\
$\geq 4.58$ & 3 & 8.1 & 0.057 \\
\hline ANC & mPFS & $95 \%$ CI & P value \\
\hline$<4.58$ & 7 & $4.4-9.6$ & \\
$\geq 4.58$ & 5 & $4.4-5.7$ & $0.024^{*}$ \\
\hline ANC & mOS & $95 \% \mathbf{C l}$ & P value \\
\hline$<4.58$ & 17.6 & $12.3-22.9$ & \\
$\geq 4.58$ & 7.3 & $4.7-10.0$ & $<0.00 I^{*}$ \\
\hline
\end{tabular}

Notes: The response to anlotinib between different groups were compared by Pearson $\chi 2$. The Log rank test was used for univariate analyses of PFS and OS. * $p<0.05$.

cancer patients. An analysis by the Japan Multinational Trial Organization reported that the ANC was higher in lung cancer patients than in healthy subjects, and the level of pretreatment ANC was an independent prognostic factor for advanced lung cancer. ${ }^{21}$ Studies conducted in China and other countries have concluded that patients with abnormally increased peripheral blood granulocyte levels had a shorter tumor-free survival time after lung surgery compared to patients with normal levels. Zer et al also reported that lower ANC levels during treatment was associated with the response to PD-1 axis inhibitors. ${ }^{22}$ One possible explanation

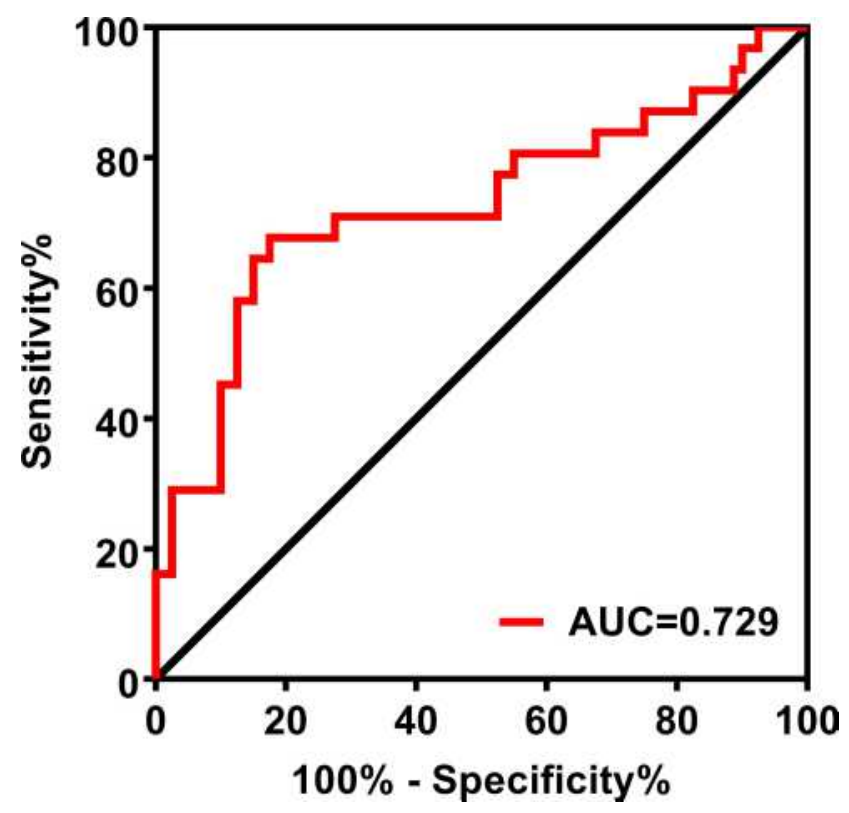

Figure $4 \mathrm{ROC}$ curve of the ANC for predicting 10-month survival of patients with advanced lung cancer. The area under the curve (AUC) of the ROC curve was 0.729 The sensitivity and specificity were $82.5 \%$ and $67.7 \%$, respectively. 
is that leukocytosis caused by granulocyte-colony stimulating factor or granulocyte macrophage-colony stimulating factor made the lung tumors more aggressive. Another hypothesis is that the cytokines interleukin- 6 and tumor necrosis factor-alpha could also induce neutrophilia, which implies suppression of anti-tumor immunity and is indicative of a poor prognosis. ${ }^{21}$

As for anti-VEGF/VEGFR therapy, Botta et al carried out an observational retrospective study and found that a high number of circulating neutrophils as well as a high NLR were associated with a shorter PFS and OS in patients with advanced NSCLC who received bevacizumab (anti-VEGF) treatment. ${ }^{16}$ In patients with metastatic renal cell carcinoma, Keizman et al showed that high NLR was associated with worse efficacy of the VEGFR inhibitor (sunitinib). ${ }^{23}$ Our study reached a similar conclusion and found that high levels of ANC were accompanied by a lower ORR, PFS and OS in lung cancer patients treated with anlotinib. In addition to the pro-invasive and immunosuppressive effects of neutrophils mentioned above, preclinical studies have also shown that inflammatory cells derived from the bone marrow such as neutrophils play a crucial role in the resistance to antiangiogenesis therapy. ${ }^{24}$ More neutrophils in the tumor microenvironment may promote angiogenesis by releasing different pro-angiogenic factors such as VEGF, interleukin-8 and Bv8, or proteases such as matrix metalloproteinase -9 and elastases. ${ }^{25}$ Finally, anti-VEGF/VEGFR drugs (such as bevacizumab and anlotinib) may not block the angiogenic switch adequately. Therefore, the therapeutic effect of anlotinib is weaken in patients with lung cancer with relatively high ANC.

Other serological indicators such as CRP and ALC, which represent the systemic inflammatory and immune status in the tumor microenvironment, were also analyzed in our study. ${ }^{26}$ Previous studies had elucidated that CRP was inversely correlated with the presence of $\mathrm{CD}^{+}$infiltrating lymphocytes in the tumor milieu, which in turn is associated with poor prognosis in clinical settings. ${ }^{27}$ Additionally, a high peripheral blood neutrophils/lymphocyte ratio has been relative to be a risk factor for patient outcome. ${ }^{19}$ Platelets have been demonstrated to be associated with angiogenesis and tumor progression, and PLR is reportedly related to poor prognosis in NSCLC patients. ${ }^{28,29}$ None of these parameters were found to be statistically significant in our study. On one hand, it might be that the number of patients in our study was not enough to verify the phenomena; on the other hand, these parameters may only affect the prognosis of the lung tumor itself and have no effect on the therapeutic efficacy of anlotinib.
Our study has other limitations. The small number of patients in our center might not be sufficient to support the conclusion. For example, while the ORR of the high-value ANC group was $8.1 \%$ and that of the low-value group was $26.5 \%$, the difference in ORR between the two groups was insignificant. Moreover, although we included prior treatment in the multivariate analysis model, subsequent treatment following anlotinib therapy was not evaluated. Finally, regarding the prognosis of patients treated with anlotinib, there was a lack of exploration between the inflammatory parameters and safety of anlotinib, including the adverse events.

\section{Conclusion}

In summary, patients with lower ANC in the peripheral blood are more likely to be benefit from anlotinib treatment in the real world. It is believed that the elevation of ANC in patients with poor prognosis may be related to the inflammatory response in the local tumor microenvironment. However, the specific mechanism needs to be explored further to validate the results in anticipation of contributions to clinical oncology in the future.

\section{Ethics Statement}

The authors are accountable for all aspects of the work in ensuring that questions related to the accuracy or integrity of any part of the work are appropriately investigated and resolved. The study was conducted in accordance with the Declaration of Helsinki (as revised in 2013). The study was approved by institutional board of Ruijin hospital and individual consent for this retrospective analysis was waived.

\section{Acknowledgments}

Thanks for the support from Ruijin Hospital and the data management from Department of Respiratory and Critical Care Medicine.

\section{Author Contributions}

All authors made a significant contribution to the work reported, whether that is in the conception, study design, execution, acquisition of data, analysis and interpretation, or in all these areas; took part in drafting, revising or critically reviewing the article; gave final approval of the version to be published; have agreed on the journal to which the article has been submitted; and agree to be accountable for all aspects of the work. 


\section{Funding}

This work was supported by National Key R\&D Program of China [granted number 2017YFC1309701, 2017YFC1309700 and 2018YFC1311900]; National Natural Science Foundation of China [granted number 81570029]; Grant of Beijing Bethune Charitable Foundation [granted number BJ-RW2020003J] and Shanghai Key Discipline for Respiratory Diseases [granted number 2017ZZ02014].

\section{Disclosure}

The authors have no conflicts of interest to declare.

\section{References}

1. Sung H, Ferlay J, Siegel RL, et al. Global cancer statistics 2020: GLOBOCAN estimates of incidence and mortality worldwide for 36 cancers in 185 countries. CA Cancer J Clin. 2021. doi:10.3322/ caac. 21660

2. Torre LA, Siegel RL, Jemal A. Lung cancer statistics. Adv Exp Med Biol. 2016;893:1-19.

3. Nicholson AG, Chansky K, Crowley J, et al. The international association for the study of lung cancer lung cancer staging project: proposals for the revision of the clinical and pathologic staging of small cell lung cancer in the forthcoming eighth edition of the TNM classification for lung cancer. $J$ Thorac Oncol. 2016;11(3):300-311. doi:10.1016/j.jtho.2015.10.008

4. Politi K, Herbst RS. Lung cancer in the era of precision medicine. Clin Cancer Res. 2015;21(10):2213-2220. doi:10.1158/1078-0432. CCR-14-2748

5. Hall RD, Le TM, Haggstrom DE, et al. Angiogenesis inhibition as a therapeutic strategy in non-small cell lung cancer (NSCLC). Transl Lung Cancer Res. 2015;4(5):515-523. doi:10.3978/j.issn.22186751.2015.06.09

6. Li B, Wang S, Li C, et al. The kinetic changes of systemic inflammatory factors during bevacizumab treatment and its prognostic role in advanced non-small cell lung cancer patients. J Cancer. 2019;10 (21):5082-5089. doi:10.7150/jca.30478

7. Xie C, Wan X, Quan H, et al. Preclinical characterization of anlotinib, a highly potent and selective vascular endothelial growth factor receptor-2 inhibitor. Cancer Sci. 2018;109(4):1207-1219. doi:10.1111/cas. 13536

8. Han B, Li K, Zhao Y, et al. Anlotinib as a third-line therapy in patients with refractory advanced non-small-cell lung cancer: a multicentre, randomised Phase II trial (ALTER0302). $\mathrm{Br}$ $J$ Cancer. 2018;118(5):654-661. doi:10.1038/bjc.2017.478

9. Han B, Li K, Wang Q, et al. Effect of anlotinib as a third-line or further treatment on overall survival of patients with advanced non-small cell lung cancer: the ALTER 0303 Phase 3 Randomized Clinical Trial. JAMA Oncol. 2018;4(11):1569-1575. doi:10.1001/ jamaoncol.2018.3039

10. Shen G, Zheng F, Ren D, et al. Anlotinib: a novel multi-targeting tyrosine kinase inhibitor in clinical development. J Hematol Oncol. 2018;11(1):120. doi:10.1186/s13045-018-0664-7

11. Naqash AR, Stroud CRG, Butt MU, et al. Co-relation of overall survival with peripheral blood-based inflammatory biomarkers in advanced stage non-small cell lung cancer treated with anti-programmed cell death-1 therapy: results from a single institutional database. Acta Oncol. 2018;57(6):867-872. doi:10.1080/ 0284186X.2017.1415460
12. Laird BJ, Kaasa S, McMillan DC, et al. Prognostic factors in patients with advanced cancer: a comparison of clinicopathological factors and the development of an inflammation-based prognostic system. Clin Cancer Res. 2013;19(19):5456-5464. doi:10.1158/1078-0432. CCR-13-1066

13. Mei Z, Shi L, Wang B, et al. Prognostic role of pretreatment blood neutrophil-to-lymphocyte ratio in advanced cancer survivors: a systematic review and meta-analysis of 66 cohort studies. Cancer Treat Rev. 2017;58:1-13. doi:10.1016/j.ctrv.2017.05.005

14. Sun K, Chen S, Xu J, et al. The prognostic significance of the prognostic nutritional index in cancer: a systematic review and meta-analysis. J Cancer Res Clin Oncol. 2014;140(9):1537-1549. doi:10.1007/s00432-014-1714-3

15. Bagley SJ, Kothari S, Aggarwal C, et al. Pretreatment neutrophil-tolymphocyte ratio as a marker of outcomes in nivolumab-treated patients with advanced non-small-cell lung cancer. Lung Cancer. 2017;106:1-7. doi:10.1016/j.lungcan.2017.01.013

16. Botta C, Barbieri V, Ciliberto D, et al. Systemic inflammatory status at baseline predicts bevacizumab benefit in advanced non-small cell lung cancer patients. Cancer Biol Ther. 2013;14(6):469-475. doi: $10.4161 /$ cbt. 24425

17. Wu D, Nie J, Dai L, et al. Salvage treatment with anlotinib for advanced non-small cell lung cancer. Thorac Cancer. 2019;10 (7):1590-1596. doi:10.1111/1759-7714.13120

18. Chen D, Xu J, Zhao Y, et al. Prognostic value of tumor cavitation in extensive-stage small-cell lung cancer patients treated with anlotinib. $J$ Cancer Res Clin Oncol. 2020;146(2):401-406. doi:10.1007/ s00432-019-03064-1

19. Wang J, Zhao Y, Wang Q, et al. Prognostic factors of refractory NSCLC patients receiving anlotinib hydrochloride as the third- or further-line treatment. Cancer Biol Med. 2018;15(4):443-451. doi:10.20892/j.issn.2095-3941.2018.0158

20. Lecot P, Sarabi M, Pereira Abrantes M, et al. Neutrophil heterogeneity in cancer: from biology to therapies. Front Immunol. 2019;10:2155. doi:10.3389/fimmu.2019.02155

21. Teramukai S, Kitano T, Kishida Y, et al. Pretreatment neutrophil count as an independent prognostic factor in advanced non-smallcell lung cancer: an analysis of Japan Multinational Trial Organisation LC00-03. Eur J Cancer. 2009;45(11):1950-1958. doi:10.1016/j.ejca.2009.01.023

22. Zer A, Sung MR, Walia P, et al. Correlation of neutrophil to lymphocyte ratio and absolute neutrophil count with outcomes with PD-1 axis inhibitors in patients with advanced non-small-cell lung cancer. Clin Lung Cancer. 2018;19(5):426-34.e1. doi:10.1016/j. cllc.2018.04.008

23. Keizman D, Ish-Shalom M, Huang P, et al. The association of pre-treatment neutrophil to lymphocyte ratio with response rate, progression free survival and overall survival of patients treated with sunitinib for metastatic renal cell carcinoma. Eur $J$ Cancer. 2012;48(2):202-208. doi:10.1016/j.ejca.2011.09.001

24. Azam F, Mehta S, Harris AL. Mechanisms of resistance to antiangiogenesis therapy. Eur J Cancer. 2010;46(8):1323-1332. doi:10.1016/j. ejca.2010.02.020

25. Ferrara N. Role of myeloid cells in vascular endothelial growth factor-independent tumor angiogenesis. Curr Opin Hematol. 2010;17(3):219-224. doi:10.1097/MOH.0b013e3283386660

26. Simmons CP, Koinis F, Fallon MT, et al. Prognosis in advanced lung cancer-A prospective study examining key clinicopathological factors. Lung Cancer. 2015;88(3):304-309. doi:10.1016/j. lungcan.2015.03.020

27. Canna K, McArdle PA, McMillan DC, et al. The relationship between tumour T-lymphocyte infiltration, the systemic inflammatory response and survival in patients undergoing curative resection for colorectal cancer. Br J Cancer. 2005;92(4):651-654. doi:10.1038/ sj.bjc. 6602419 
28. Alexandrakis MG, Passam FH, Perisinakis K, et al. Serum proinflammatory cytokines and its relationship to clinical parameters in lung cancer patients with reactive thrombocytosis. Respir Med. 2002;96(8):553-558. doi:10.1053/rmed.2002.1328
29. Sierko E, Wojtukiewicz MZ. Platelets and angiogenesis in malignancy. Semin Thromb Hemost. 2004;30:95-108.

\section{Publish your work in this journal}

Cancer Management and Research is an international, peer-reviewed open access journal focusing on cancer research and the optimal use of preventative and integrated treatment interventions to achieve improved outcomes, enhanced survival and quality of life for the cancer patient.
The manuscript management system is completely online and includes a very quick and fair peer-review system, which is all easy to use. Visit http://www.dovepress.com/testimonials.php to read real quotes from published authors. 